\title{
Review
}

\section{A Long Way to Go: Patient Perspectives on Digital Health for Parkinson's Disease}

\author{
Sara Riggare $^{\mathrm{a}, *}$, Jon Stamford ${ }^{\mathrm{b}}$ and Maria Hägglund ${ }^{\mathrm{a}}$ \\ ${ }^{a}$ Department of Women's and Children's Health, Uppsala University, Uppsala, Sweden \\ ${ }^{\mathrm{b}}$ Gentleman Neuroscientist and Independent Parkinson's Patient Advocate, UK
}

Accepted 10 February 2021

Pre-press 27 February 2021

\begin{abstract}
Digital health promises to improve healthcare, health, and wellness through the use of digital technologies. The purpose of this commentary is to review and discuss the field of digital health for Parkinson's disease (PD) focusing on the needs, expectations, and wishes of people with $\mathrm{PD}(\mathrm{PwP})$. Our analysis shows that $\mathrm{PwP}$ want to use digital technologies to actively manage the full complexity of living with PD on an individual level, including the unpredictability and variability of the condition. Current digital health projects focusing on PD, however, does not live up to the expectations of PwP. We conclude that for digital health to reach its full potential, the right of PwP to access their own data needs to be recognised, PwP should routinely receive personalised feedback based on their data, and active involvement of PwP as an equal partner in digital health development needs to be the norm.
\end{abstract}

Keywords: Digital health, patient perspectives, digital technology, patient empowerment

\section{INTRODUCTION}

Digital health promises to improve healthcare, health and wellness through the use of digital technologies. Parkinson's disease (PD) with its abundance of symptoms and treatment side effects as well as highly individualised manifestations and treatment combinations has been identified as a suitable model disease for technology-based improvements [1-3].

Today, the field of telehealth for PD can be considered established and reasonably well researched [4, 5]. The field has also been given a boost by the ongoing covid-19 pandemic [6]. Furthermore, technology is used in delivery of physiotherapy and similar [7-9]. Although helpful to many, we will not focus on these fields, but rather explore more innovative and PD specific digital health solutions.

\footnotetext{
*Correspondence to: Sara Riggare, Department of Women's and Children's Health, Uppsala University, Dag Hammarskjölds väg 14B, 1 tr, 75237 Uppsala, Sweden. Tel.: +46 70 3565104; E-mail: sara.riggare@kbh.uu.se.
}

The rapid technological evolution over the past two decades is enabling new models of knowledge exchange. For instance, the number of smartphones in the world today is estimated to be 3.5 billion (the world's population is 7.8 billion) and is forecasted to keep increasing [10]. This means that an ever-increasing number of people have access to the world's collective knowledge, literally at our fingertips. What does this mean for digital health in PD?

Even though patient engagement has been suggested to have clinical effects on levels comparable to "blockbuster drugs" [11], the patient perspective of digital health is rarely discussed, it has even been reported that physicians are discouraging patients from using digital solutions [12]. The purpose of this commentary is therefore to review and discuss the field of digital health for PD from a patient perspective, primarily focusing on the needs, expectations, and wishes of people with PD (PwP). Two of the authors are $\mathrm{PwP}$ with substantial experience of digital health for PD and their personal perspectives will be incorporated throughout the article. 


\section{WHAT DO PWP WANT FROM DIGITAL HEALTH?}

Management of any chronic condition is a mix of healthcare and selfcare. For PD, as emphasised by the ongoing covid-19 pandemic, selfcare is key to successful management [13]. What do PwP hope for and expect from digital technologies for their selfcare? Technology, including online tools and services have been identified as having potential to improve health and well-being for PwP [14]. In general, PwP often have access to and feel comfortable using computers, mobile phones and the Internet [15, 16], although, similarly to the general population, the use of smartphone apps is lower among older PwP compared to younger [15]. Several recent studies report that PwP are optimistic that digital technologies will enable them to improve their abilities to successfully manage the progressive nature of PD [17-19]. Our review of the literature shows that PwP want to use digital technologies for:

- Actively managing the full complexity of living with PD on an individual level, including the unpredictability and variability of the condition [17-21].

- Tracking functionality and symptoms (motor and non-motor) in relation to medications (types, doses, and timings), stress, sleep, exercise, and contextual factors [17-21].

- Collaborating with their medical team in decisions on symptoms, problem areas, treatments, short and long term management [17-21].

- Finding information, knowledge, and social support [22-25].

Active management of the full complexity of living with PD requires access to information. It is wellknown that a lot of people in the general public are finding health information online and one study reported that $75 \%$ of $\mathrm{PwP}$ access disease-related information online [15]. In a different study, 36\% of PwP identified the Internet as their main source of PD-specific knowledge [16]. The accuracy, relevance, and quality of the information online can however vary [26] and concerns have been raised both by clinicians and $\mathrm{PwP}$ of the difficulty for $\mathrm{PwP}$ to separate "hope from hype" [27]. One example is a study of how DBS is represented in videos on Youtube; as "a technological fix", while the more complex impacts of the procedure are not elaborated on [28]. This can lead to unrealistic expectations from $\mathrm{PwP}$ on what can be achieved.
Self-tracking is "a process of deliberately collecting and structuring observations about one's own life" [29] and it can be used by PwP both in collaboration with clinicians and researchers, and on their own, as patient-initiated self-tracking. To the best of our knowledge, the only peer-reviewed academic work on patient-initiated self-tracking in PD has been conducted by the first author of this paper (SR) and includes two single subject studies where SR used herself as the research participant. In the first study, SR explored how the effects of her PD medication varied across the day by capturing finger tapping performance with a smartphone app [30]. In the second study, the effect of nicotine from an e-cigarette on levodopa-induced dyskinesias was examined [31]. The main conclusion from these two studies is that self-observation and symptom tracking potentially can enable PwP to better understand their own personal condition and to improve treatment, both with and without clinical support. Evaluation of a selfmanagement programme for PwP and care partners confirms that such effects are possible [32].

Daily feedback to PwP of their data from wearable sensor technology has been shown to elicit positive effects on individual mobility compared to PwP wearing the sensors without feedback [33].

\section{CURRENT STATE OF DIGITAL HEALTH FOR PD}

Digital technologies have been used for more than a decade to assist clinicians in assessments of PD. Set against this backdrop, to what extent do current digital technologies match patient expectations or hopes? Below we will present a few illustrative examples from the clinical/research communities in PD and consumer device industry.

Over the last few years, there have been a number of initiatives and projects using wearable technology to track different aspects of PD. To date, only a few are generally available, and then mostly to clinicians only. One such example is the Personal Kinetigraph (PKG); a device worn on the wrist, collecting data on movements (tremor, bradykinesia, and dyskinesia) and providing reminders for medication intakes. The patient receives a device, pre-programmed by a clinician and wears it for six consecutive days. A report is generated and used by the physician in discussions with the patient. A recent study reported that physicians found that using the device could improve patient dialogue and that patients found that it could 
provide valuable impact on their care [34]. The PKG cannot be acquired directly by $\mathrm{PwP}$ and the report that is generated is also not available to PwP.

A prototype version of a wearable device aiming to improve monitoring of PD on/off states using a single sensor was developed in an EU-funded project between 2011 and 2015. The system, called REMPARK, consists of a sensor worn in a belt and a smartphone app and is designed to record the movement of PwP and provide cues to improve walking as well as nudges to fill out questionnaires on nonmotor symptoms. A multi-center five day validation study indicated that the system was able to correctly detect on/off state in a study of $33 \mathrm{PwP}$, comparing data from the sensor with PwP self-reported on/off states [35]. The system enables clinicians to monitor PwP online, PwP do not have access to the data generated.

A smartphone app for PD that has received a lot of attention is mPower. It was launched as part of Apple Research Kit in March 2015 with the aim of a better understanding of the variations of PD and potential modulators as well as providing real-time feedback to the participants [36]. Although the mPower app was initially downloaded 48,104 times, only $0.3 \%$ $(n=150)$ of users were PwP who contributed data on five separate days or more [37]. Publications using mPower data show interesting results but as far as we can understand, the intended real-time feedback to participants has not yet been implemented.

Devices not specifically intended for PD may also be useful. Spire ${ }^{\circledR}$ is a wearable device for tracking breathing, heart rate and activity. It can be acquired by clinicians and is used for monitoring patients, mainly with COPD. A small $(n=13)$ randomised study evaluating the device in the context of mindfulness training for anxiety in PD reported challenges with study recruitment and retention, acceptability of the device, and technical difficulties [38]. The conclusion was that in future studies, PwP need to be involved already in the early planning stages.

Parkinson@Home is a recent project with the objective of investigating the use of technology for PwP in their daily lives by using a smartwatch together with a smartphone and an app combined with a cloud storage environment [39]. A recently published validation study $(n=42)$ demonstrated that using a single sensor to analyse gait in the homes of PwP is feasible [40].

Consumer devices may also be of interest. A device intended for PD that has been discussed a lot among PwP during the last few years is the "Emma Watch", a vibrating device worn on the wrist intended to compensate for hand tremor. The project was funded by Microsoft and involved a team lead by an innovation director at the company and a PwP with young onset [41]. As can be seen from the +26000 Google search hits and +400000 views on Youtube, the project received worldwide attention, yet there is no indication that the device has been developed further since 2017.

\section{DISCUSSION}

Digital health holds significant potential of transforming health, healthcare, and research. We have reviewed the literature with a specific focus on the needs, expectations, and wishes of people with PD (PwP) [17-25]. In Table 1 the future development aims of digital technologies according to our analysis of the aspirations of PwP are summarised.

When comparing these development aims with the solutions presented in the previous section, it is apparent that the field of digital health in PD has not come very far. Consumer products have so far also failed to make it beyond laboratory or pilot settings. PwP cannot access the generated data in any of the solutions presented above which means that PwP cannot themselves learn directly from the data they generate. To date, the majority of studies conducted in digital health for PD focus on assessments in the clinic despite the fact that the overwhelming majority of PwP's time is spent outside of the clinic [42]. Notable exceptions are the mPower smartphone app and the Parkinson@Home study, which are both collecting data from PwP in their everyday lives. The two projects have however yet to deliver tangible results for the larger PwP community. Publications using mPower data show interesting results but as far as we can understand, the intended real-time feedback to participants has not yet been implemented, which could be one explanation for the very high attrition rates. Another important issue is the lack of personalised feedback to PwP since meaningful feedback to PwP has been shown to increase motivation [33].

Conventional studies often attempt to identify markers to predict the onset and progression of PD by observing and collecting data from individuals and draw conclusions on a group level. This is of course of interest to PwP but considering the extreme individuality of $\mathrm{PD}$, such an effort may take a very long time to produce any tangible results available to $\mathrm{PwP}$ or even fail completely. Of higher priority to $\mathrm{PwP}$ is 
Table 1

Future development aims of digital technologies according to aspirations of PwP

\begin{tabular}{|c|c|}
\hline Domain & Future development aims \\
\hline \multicolumn{2}{|c|}{ To meet the aspirations of $P w P, \ldots$ digital technologies should } \\
\hline Availability & ... be available to PwP directly, and not only via clinicians. \\
\hline \multirow[t]{2}{*}{ Functionality } & ... work for both motor and non-motor symptoms. \\
\hline & $\begin{array}{l}\text {.. support both short term hour-by-hour management of medication } \\
\text { and long-term monitoring of year-by-year progression of disease. } \\
\ldots \text { support collaboration between PwP and their medical team. }\end{array}$ \\
\hline \multirow[t]{2}{*}{ Data } & ... provide PwP with access to data collected. \\
\hline & ... give PwP personalised feedback based on the data. \\
\hline
\end{tabular}

probably to use their own data to improve their own PD.

The development aims in Table 1 can be seen as building blocks for personal science, which is "the practice of using empirical methods to explore personal questions" [29, 43]. Personal science and patient-initiated self-tracking offer a framework for structured self-observation that can be supported by the use of technology but can also be applied without digital tools.

\section{THE PAST, PRESENT, AND FUTURE OF DIGITAL HEALTH FOR PD}

We have summarised our view of how the roles of PwP, clinicians and researchers in the design and use of digital health for PD are evolving in Fig. 1. In the past, PWP involvement in digital health development has been small and mainly in the context of technological research. This resulted in many "solutions looking for a problem" rather than developing solutions based on actual needs of PwP. At present, there is more interaction generally and it is with both

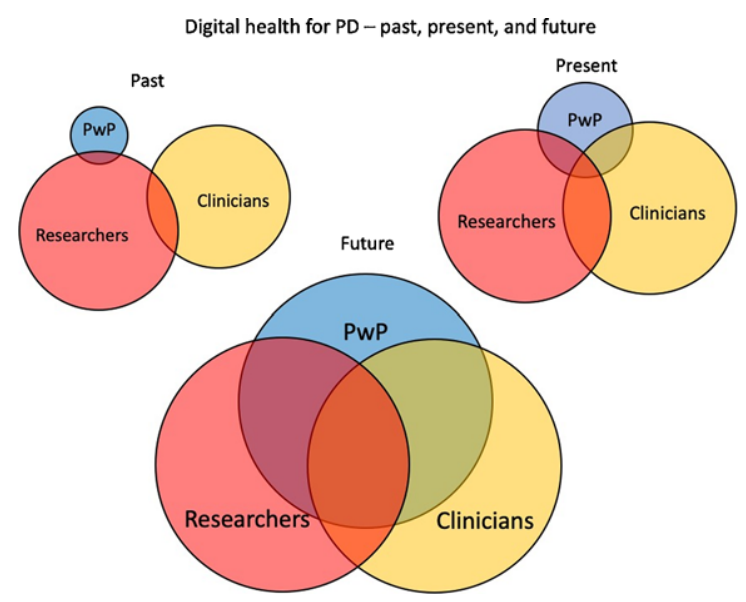

Fig. 1. The past, present, and future of digital health for PD. researchers and clinicians, although the role of $\mathrm{PwP}$ is often significantly smaller than that of researchers and clinicians. One explanation can be that clinicians are not always comfortable with the way PwP use or want to use technology [21].

In the future it is to be hoped that patient involvement will increase substantially and in equal partnership with researchers and clinicians.

By weighing together our review of the literature with our personal experiences, we conclude that for digital health for PD to reach its full potential, a number of challenges need to be addressed:

1) The right of PwP to access their own data needs to be recognised

2) PwP should routinely receive personalised feedback based on their data, and

3) Active involvement of PwP as an equal partner in digital health development needs to be the norm, and if necessary mandated and/or incentivised.

\section{CONFLICT OF INTEREST}

The authors have no conflicts of interest to report.

\section{REFERENCES}

[1] Klucken J, Kruger R, Schmidt P, Bloem BR (2018) Management of Parkinson's disease 20 years from now: Towards digital health pathways. J Parkinsons Dis 8, S85-S94.

[2] Bloem B, Henderson E, Dorsey R, Okun M, Okubadejo N, Chan P, Andrejack J, Darweesh S, Munneke M (2020) Integrated and patient-centred management of Parkinson's disease: A network model for reshaping chronic neurological care. Lancet Neurol 4422, 1-12.

[3] Hansen C, Sanchez-Ferro A, Maetzler W (2018) How mobile health technology and electronic health records will change care of patients with Parkinson's disease. J Parkinsons Dis 8, S41-S45.

[4] Beck CA, Beran DB, Biglan KM, Boyd CM, Dorsey ER, Schmidt PN, Simone R, Willis AW, Galifianakis NB, Katz M, Tanner CM, Dodenhoff K, Aldred J, Carter J, Fraser A, Jimenez-Shahed J, Hunter C, Spindler M, Reichwein S, Mari Z, Dunlop B, Morgan JC, McLane D, Hickey P, Gauger L, Richard IH, Mejia NI, Bwala G, Nance M, Shih 
LC, Singer C, Vargas-Parra S, Zadikoff C, Okon N, Feigin A, Ayan J, Vaughan C, Pahwa R, Dhall R, Hassan A, DeMello S, Riggare SS, Wicks P, Achey MA, Elson MJ, Goldenthal S, Keenan HT, Korn R, Schwarz H, Sharma S, Stevenson EA, Zhu W (2017) National randomized controlled trial of virtual house calls for Parkinson disease. Neurology 89, 1152-1161.

[5] Kraepelien M, Schibbye R, Månsson K, Sundström C, Riggare S, Andersson G, Lindefors N, Svenningsson P, Kaldo V (2020) Individually tailored Internet-based cognitivebehavioral therapy for daily functioning in patients with Parkinson's disease: A randomized controlled trial. $J$ Parkinsons Dis 10, 653-664.

[6] Dorsey ER, Okun MS, Bloem BR (2020) Care, convenience, comfort, confidentiality, and contagion: The $5 \mathrm{C}$ 's that will shape the future of telemedicine. J Parkinsons Dis 10, 893897.

[7] Chamorro-Moriana G, Moreno AJ, Sevillano JL (2018) Technology-based feedback and its efficacy in improving gait parameters in patients with abnormal gait: A systematic review. Sensors (Switzerland) 18, 142.

[8] Theodoros D, Aldridge D, Hill AJ, Russell T (2019) Technology-enabled management of communication and swallowing disorders in Parkinson's disease: A systematic scoping review. Int $J$ Lang Commun Disord 54, 170-188.

[9] Lei C, Sunzi K, Dai F, Liu X, Wang Y, Zhang B, He L, Ju M (2019) Effects of virtual reality rehabilitation training on gait and balance in patients with Parkinson's disease: A systematic review. PLoS One 14, e0224819.

[10] Statista.com, Number of smartphone users worldwide from 2016 to 2021, Accessed on October 14, 2020, https://www.statista.com/statistics/330695/number-ofsmartphone-users-worldwide/.

[11] Dentzer S (2013) Rx for the "Blockbuster Drug" of patient engagement. Health Aff 32, 202.

[12] Roper L, Jorm C (2017) Please leave your phone outside: Policymakers and medical app providers are encouraging patient participation, but doctors in the consult room are not. BMJ Innov 3, 65-70.

[13] Helmich RC, Bloem BR (2020) The impact of the COVID19 pandemic on Parkinson's disease: Hidden sorrows and emerging opportunities. J Parkinsons Dis 10, 351-354.

[14] Stamford J, Schmidt P, Friedl K (2015) What engineering technology could do for quality of life in Parkinson's disease: A review of current needs and opportunities. IEEE $J$ Biomed Health Inform 19, 1862-1872.

[15] Marxreiter F, Buttler U, Gassner H, Gandor F, Gladow T, Eskofier B, Winkler J, Ebersbach G, Klucken J (2020) The use of digital technology and media in German Parkinson's disease patients. J Parkinsons Dis 10, 717-727.

[16] Riggare S, Höglund PJ, Hvitfeldt Forsberg H, Eftimovska E, Svenningsson P, Hägglund M (2017) Patients are doing it for themselves: A survey on disease-specific knowledge acquisition among people with Parkinson's disease in Sweden. Health Informatics $J \mathbf{2 5}, 91-105$.

[17] Mishra SR, Klasnja P, Woodburn JM, Hekler EB, Omberg L, Kellen M, Mangravite L (2019) Supporting coping with Parkinson's disease through self-tracking. 2019 CHI Conf Hum Factors Comput Syst CHI, pp. 1-16.

[18] Riggare S, Duncan TS, Hvitfeldt H, Hägglund M (2019) "You have to know why you're doing this": A mixed methods study of the benefits and burdens of self-tracking in Parkinson's disease. BMC Med Inform Decis Mak 19, 175 .
[19] Memedi M, Tshering G, Fogelberg M, Jusufi I, Kolkowska E, Klein G (2018) An interface for IoT: Feeding back healthrelated data to Parkinson's disease patients. J Sens Actuator Networks 7, 14.

[20] Nunes F, Andersen T, Fitzpatrick G (2019) The agency of patients and carers in medical care and self-care technologies for interacting with doctors. Health Informatics $J \mathbf{2 5}$, 330-349.

[21] Ozanne A, Johansson D, Hällgren Graneheim U, Malmgren K, Bergquist F, Alt Murphy M (2018) Wearables in epilepsy and Parkinson's disease-A focus group study. Acta Neurol Scand 137, 188-194.

[22] Riggare S (2018) E-patients hold key to the future of healthcare. BMJ 846, k846.

[23] Shapira AL, Handzel R, Korczyn AD (2017) The lived experience of Parkinson's disease: A content analysis of parkinson's patients' blogs. Isr Med Assoc J 19, 685-690.

[24] Martínez-Pérez B, Bargiela-Flórez B, López-Coronado M, De La Torre-Díez I, Rodrigues JJPC (2015) Content analysis of neurodegenerative and mental diseases social groups. Health Informatics $J \mathbf{2 1}$, 267-283.

[25] Visser LM, Bleijenbergh IL, Benschop YWM, Van Riel ACR, Bloem BR (2016) Do online communities change power processes in healthcare? Using case studies to examine the use of online health communities by patients with Parkinson's disease. BMJ Open 6, e012110.

[26] Kim R, Kim HJ, Jeon B (2018) The good, the bad, and the ugly of medical information on the Internet. Mov Disord 33, 754-757.

[27] Robledo I, Jankovic J (2017) Media hype: Patient and scientific perspectives on misleading medical news. Mov Disord 32, 1319-1323.

[28] Gardner J, Warren N, Addison C, Samuel G (2019) Persuasive bodies: Testimonies of deep brain stimulation and Parkinson's on YouTube. Soc Sci Med 222, 44-51.

[29] Groot M De, Wolf GI (2020) A conceptual framework for personal science. Front Comput Sci 2, 1-5.

[30] Riggare S, Hägglund M (2018) Precision medicine in Parkinson's disease - exploring patient-initiated selftracking. J Parkinsons Dis 8, 441-446.

[31] Riggare S, Unruh KT, Sturr J, Domingos J, Stamford JA, Svenningsson P, Hägglund M (2017) Patient-driven N-of-1 in Parkinson's disease: Lessons learned from a placebocontrolled study of the effect of nicotine on dyskinesia. Methods Inf Med 56, 123-128.

[32] Hellqvist C, Berterö C, Hagell P, Dizdar N, Sund-Levander M (2020) Effects of self-management education for persons with Parkinson's disease and their care partners: A qualitative observational study in clinical care. Nurs Heal Sci 22, 741-748.

[33] Uem JMT Van, Maier KS, Hucker S, Scheck O, Hobert MA, Santos AT (2016) Twelve-week sensor assessment in Parkinson's disease: Impact on quality of life. Mov Disord 31, 1337-1338.

[34] Joshi R, Bronstein JM, Keener A, Alcazar J, Yang DD, Joshi M, Hermanowicz N (2019) PKG movement recording system use shows promise in routine clinical care of patients with Parkinson's disease. Front Neurol 10, 1-11.

[35] Bayés Á, Samá A, Prats A, Pérez-López C, Crespo-Maraver M, Moreno JM, Alcaine S, Rodriguez-Molinero A, Mestre B, Quispe P, de Barros AC, Castro R, Costa A, Annicchiarico R, Browne P, Counihan T, Lewy H, Vainstein G, Quinlan LR, Sweeney D, ÓLaighin G, Rovira J, Rodrigue z-Martin D, Cabestany J (2018) A “HOLTER" for Parkinson's disease: 
Validation of the ability to detect on-off states using the REMPARK system. Gait Posture 59, 1-6.

[36] Dorsey ER, Chan Y-F, McConnell MV, Shaw SY, Trister AD, Friend SH (2017) The use of smartphones for health research. Acad Med 92, 157-160.

[37] Bot BM, Suver C, Neto EC, Kellen M, Klein A, Bare C, Doerr M, Pratap A, Wilbanks J, Dorsey ER, Friend SH, Trister AD (2016) The mPower study, Parkinson disease mobile data collected using ResearchKit. Sci Data 3, 160011.

[38] Lingaiah A, Mohanty D, Marchbank J, Jacob AE, Castellanos RL, Holiday V, Espay AJ, LaFaver K (2020) Improving anxiety in Parkinson's disease: A cautionary tale about mobile health technologies. Parkinsonism Relat Disord 73, 50-51.

[39] Silva de Lima AL, Hahn T, de Vries NM, Cohen E, Bataille L, Little MA, Baldus H, Bloem BR, Faber MJ (2016) Largescale wearable sensor deployment in Parkinson's Patients: The Parkinson@Home Study Protocol. JMIR Res Protoc 5, e172.
[40] Evers LJ, Raykov YP, Krijthe JH, Silva de Lima AL, Badawy R, Claes K, Heskes TM, Little MA, Meinders MJ, Bloem BR (2020) Real-life gait performance as a digital biomarker for motor fluctuations: The Parkinson@Home validation study. J Med Internet Res 22, 1-18.

[41] Microsoft, Project Emma, Last updated 2017, Accessed on October 12, 2020, https://www.microsoft.com/enus/research/project/project-emma/.

[42] Dorsey ER, Omberg L, Waddell E, Adams JL, Adams R, Ali MR, Amodeo K, Arky A, Augustine EF, Dinesh K, Hoque ME, Glidden AM, Jensen-Roberts S, Kabelac Z, Katabi D, Kieburtz K, Kinel DR, Little MA, Lizarraga KJ, Myers T, Riggare S, Rosero SZ, Saria S, Schifitto G, Schneider RB, Sharma G, Shoulson I, Stevenson EA, Tarolli CG, Luo J, McDermott MP (2020) Deep phenotyping of Parkinson's disease. J Parkinsons Dis 10, 855-873.

[43] Heyen NB (2019) From self-tracking to self-expertise: The production of self-related knowledge by doing personal science. Public Underst Sci 29, 124-138. 